\title{
Actividad in vitro de niveles séricos y urinarios de amoxicilina y amoxicilina-sulbactam sobre 820 cepas de Escherichia coli aisladas de infecciones urinarias bajas extrahospitalarias. Estudio sudamericano
}

JOSÉ MARÍA CASELLAS ${ }^{1 *}$, MATÍAS VISSER ${ }^{1}$, BÁRBARA COCO ${ }^{1}$, NATALIA MAC DOUGALL $^{1}$, HUGO COHEN S. ${ }^{2}$, JORGE SOUTRIC ${ }^{3}$, HÉCTOR ARENOSO ${ }^{3}$ y GRUPO COLABORATIVO LATINOAMERICANO*

\section{In vitro activity at serum and urinary levels of amoxicillin and amoxicillin- sulbactam against 820 Escherichia coli strains isolated from community acquired urinary tract infections. South American collaborative study}

We previously showed urinary activity against Escherichia coli both after oral amoxicillinsulbactam and sulbactam alone. A study was performed in 10 laboratories from 8 countries to determine the etiology of community acquired lower urinary tract infections (CALUTI) in patients aged 3-to 7 years in South America. Each laboratory sent data from 100 consecutive isolates including susceptibility to amoxicillin and amoxicillin-sulbactam by agar diffusion and also sent 20 consecutive E. coli isolates resistant (R) to amoxicillin-sulbactam to CEA reference laboratory, were MICs were determined for amoxicillin, amoxicillin-sulbactam (2/1) and sulbactam. Urines from 12 volunteers who received a single oral dose 500/500 mg amoxicillin-sulbactam were collected at 0-2; 2-4 and 4-6 h and urinary inhibitory titers (UIT) were determined against $5 \mathrm{R}$ and 1 susceptible (S) isolates from each center. Levels of amoxicillin and sulbactam were determined. Results: Etiology: E. coli 820/1000 $(82 \%)$ was prevalent; $P$. mirabilis and $K$. pneumoniae (4,3\% each), S. saprophyticus 4,1\% other 5,3\%. Diffusion test: $59,4 \%$ E. coli were R to amoxicillin, 16,9 intermediate (I) and 23,7 S whereas results for amoxicillin-sulbactam were $28 \% \mathrm{R} ; 19,2$ I and 52,8 S. MICs: 102 E. coli $\mathrm{R}$ to amoxicillinsulbactam were studied; MIC90s $(\mu \mathrm{g} / \mathrm{ml})$ were the following: amoxicillin > 2.048; amoxicillinsulbactam 256/128 and sulbactam alone 128. UITs: ranged from $>1 / 32$ at $2 \mathrm{~h}$ dose; $1 / 16-1 / 4 \mathrm{~h}$ and $1 /$ $4-1 / 2$ at $6 \mathrm{~h}$ against all the isolates. Antibacterial urine levels $(\mu \mathrm{g} / \mathrm{ml})$ obtained for amoxicillin and sulbactam respectively were at $2 \mathrm{~h} 1.414$ and 1.904; at $4 \mathrm{~h} 691$ and 1.257 and at $6 \mathrm{~h} 462$ and 641 . Our results confirm the prevalence of E. coli and explain discrepancies between resistance shown by agar diffusion test and clinical success observed in the treatment of CALUTI.

Key words: Urinary tract infections; amoxicillin-sulbactam; Latin America.

\footnotetext{
Centro de Estudios en Antimicrobianos (CEA), Bs. As., Argentina

Hospital Manuel Belgrano, San Martín, B A, Argentina

Departamento Médico de Laboratorios Bagó, Argentina
}

Estudio financiado por Laboratorios Bagó.

*Grupo Colaborativo Latinoamericano: René Botelho, Hospital de Clínicas, La Paz, Bolivia; Lidia Carvajal, Hospital de Niños, Córdoba, Argentina; Catalina Culasso, Hospital de Niños, Córdoba, Argentina; Roxana De La Vega, Hospital de Clínicas, La Paz, Bolivia; M. del Carmen Espínola, Hospital de Previsión Social, Asunción, Paraguay; Patricia García, Pontificia Universidad Católica de Chile; Humberto Guerra, Universidad Peruana Cayetano Heredia, Lima, Perú; Damián Ingaramo, IDIMYC, Rosario, Argentina; Walter Pedreira, Universidad Nacional, Montevideo, Uruguay; Adriana Reis, Universidade Federal de Sao Paulo, Brasil; Hélio Sader, Universidade Federal de Sao Paulo, Brasil; J. Pablo Scappini, IDIMYC, Rosario, Argentina; Gabriela Tomé, LACCIM, Martínez, BA, Argentina y Jeannette Zurita, Universidad Nacional, Quito, Ecuador.

Recibido: 27 junio 2002

Aceptado: 18 diciembre 2002 


\section{Introducción}

Las infecciones urinarias (IU), bajas e inclusive muchas IU altas no bacteriémicas que ocurren en niños y en pacientes del sexo femenino sin obstrucciones de las vías urinarias, dependen para su tratamiento de los niveles urinarios de los antibacterianos, y no necesariamente de sus niveles séricos ${ }^{1}$.

Escherichia coli es la especie prevalente en las IU no complicadas adquiridas en la comunidad en ambos sexos y en todos los grupos etarios.

Amoxicilina-sulbactam es un antimicrobiano que combina dos moléculas $\beta$-lactámicas: amoxicilina, y un inhibidor de $\beta$-lactamasas: sulbactam, que es capaz de bloquear la producción, a niveles normales, de la $\beta$-lactamasa plasmídica TEM-1 responsable de más de $95 \%$ de ocurrencia de resistencia en cepas de Escherichia coli aisladas de pacientes en la comunidad ${ }^{2}$. Con el agregado de sulbactam y otros inhibidores de $\beta$-lactamasas, la resistencia a aminopenicilinas en las cepas de E. coli que varía entre 60 y $80 \%$ en Sudamérica, desciende a niveles que oscilan entre 30 y $45 \%{ }^{3}$. Ello implica que, teóricamente, en ese porcentaje, deberían fracasar los tratamientos con amoxicilinasulbactam de IU bajas causadas por E. coli.

Sin embargo, basados en investigaciones que previamente habíamos efectuado ${ }^{4}$, Bantar et al. demostraron ${ }^{5}$ en estudios ex-vivo en voluntarios, que amoxicilina-sulbactam alcanza un título inhibitorio de la orina (TIO) suficiente para erradicar cepas hiperproductoras de la $\beta$-lactamasa TEM-1. Más adelante, estudios realizados por nuestro grupo de trabajo demostraron que la actividad inhibitoria de amoxicilina-sulbactam y de amoxicilinaclavulanato es también debida a la acción intrínseca del inhibidor solo, independientemente de su combinación con aminopenicilinas. La concentración de sulbactam que se alcanza en orina con las combinaciones comercializadas de amoxicilinasulbactam supera los $1.000 \mu \mathrm{g} / \mathrm{ml}$, la que es considerablemente mayor que las CIMs que hemos observado a sulbactam de todas las cepas de E. coli ensayadas hasta ahora en la Argentina y que oscilan entre 32 y $256 \mu \mathrm{g} / \mathrm{ml}^{6}$.

En base a estos estudios postulamos que los resultados de los antibiogramas efectuados con cepas aisladas de orina en pacientes que desarrollan IU bajas no complicadas, no bacteriémicas, comunitarias (IUBNCC), muestran resultados falaces cuando expresan resistencia ya que los puntos de corte que se utilizan, establecidos para concentraciones séricas, no se corresponden con las concentraciones de amoxicilina ni de sulbactam esperables en la orina. Para ello decidimos efec- tuar un estudio multicéntrico en Sudamérica destinado a los siguientes objetivos:

- Determinar en 10 centros sudamericanos, la prevalencia de las especies bacterianas responsables de IU bajas, extrahospitalarias, no complicadas, no bacteriémicas, de orinas consecutivamente obtenidas de niños y adultos, y su susceptibilidad a amoxicilina y amoxicilinasulbactam por el método de discos convencional.

- Determinar en 20 cepas consideradas resistentes a amoxicilina-sulbactam, provenientes de cada centro, el valor de la CIM por macrodilución de amoxicilina, sulbactam y la combinación amoxicilina-sulbactam.

- Correlacionar los resultados obtenidos por el método de discos con los valores de CIM de cada fármaco y la concentración de los mismos alcanzables en la orina.

- Determinar el TIO en voluntarios luego de la ingestión de 500/500 mg de amoxicilinasulbactam, así como las concentraciones de amoxicilina y sulbactam alcanzadas en las orinas emitidas.

\section{Material y Método}

Centros participantes: Argentina: Hospital de Niños, Córdoba. IDIMYC, Rosario. LACCIM, Martínez, B A.; Bolivia: Hospital de Clínicas, La Paz; Brasil: Universidade Federal, Sao Paulo; Chile: Pontificia Universidad Católica de Chile; Ecuador: Universidad Nacional, Quito; Paraguay: Hospital de Previsión Social, Asunción; Perú: Universidad Peruana Cayetano Heredia, Lima; Uruguay: Universidad Nacional, Montevideo.

Cepas: Cada centro recuperó en forma sucesiva 100 aislamientos provenientes de la orina de pacientes ambulatorios de ambos sexos, mayores de 3 años, que presentaban IUBNCC. Las orinas debían presentar desarrollo de más de $10.000 \mathrm{ufc} / \mathrm{ml}$ de un cultivo monomicrobiano, coincidente con un sedimento urinario en el que se observaran más de 10 leucocitos por campo $(400 \mathrm{x})$ para ser incluidas en el estudio.

Las cepas fueron identificadas a nivel de especie de acuerdo a las recomendaciones de la American Society for Microbiology ${ }^{7}$.

Se remitieron al centro coordinador, Centro de Estudios en Antimicrobianos (CEA):

- Veinte cepas, sucesivamente aisladas, resistentes a amoxicilina-sulbactam de acuerdo a los criterios de NCCLS para ampicilinasulbactam $^{8}$ que fueron inoculadas en tubos Eppendorf conteniendo agar blando Columbia 
(Biokar, Francia). Aquellos centros que no recuperaron 20 cepas de E. coli resistentes entre las 100 cepas estudiadas, prosiguieron estudiando cepas sucesivas hasta completar el número de 20 cepas resistentes. Por otra parte, y con el objetivo de disponer de cepas testigo, cada centro remitió 5 cepas consideradas susceptibles a amoxicilina-sulbactam.

- Una planilla con la información sobre datos demográficos del paciente, cantidad de leucocitos por campo, recuento de colonias, identificación bacteriana, y susceptibilidad a amoxicilina y amoxicilina-sulbactam por el método de discos.

\section{Estudios efectuados por el centro coordinador.}

- Las cepas recibidas fueron resembradas y se comprobó su pureza y correcta identificación.

- Se efectuó la determinación de la CIM de amoxicilina, sulbactam y la combinación amoxicilina-sulbactam.

- Se determinaron los niveles urinarios de amoxicilina y de sulbactam en la orina de los voluntarios.

- Se determinó el TIO de los voluntarios a cepas seleccionadas de E. coli resistentes y susceptibles a amoxicilina-sulbactam.

\section{Pruebas de susceptibilidad a los antimicro- bianos.}

- Difusión: Se utilizaron discos de un mismo lote de amoxicilina $10 \mu \mathrm{g}$ y amoxicilinasulbactam 10/10 $\mu$ g (Medicatec, Argentina), y fueron controlados en el CEA de acuerdo a las recomendaciones de la NCCLS M2-A7 ${ }^{9}$ para ampicilina-sulbactam 10/10 con cepas de E. coli ATCC 25922 y E. coli ATCC 35218. En Chile, por razones operativas se utilizaron discos preparados localmente, pero controlados en el CEA. Cada laboratorio usó el mismo lote de agar Mueller Hinton (Biokar, Francia).

- Dilución: Las CIM para amoxicilina, sulbactam y la combinación amoxicilina-sulbactam fueron determinadas en el CEA en forma duplicada usando el método de macrodilución en agar, de acuerdo a las recomendaciones de NCCLS M100-S2 ${ }^{8}$. Se utilizó un aparato replicador de Steers para la inoculación. Amoxicilina-sulbactam fue utilizada en relación $2: 1$. Se utilizó amoxicilina sódica y sulbactam sódico como fármaco puro con potencia valorada provistos por Laboratorios Bagó Argentina. Se ensayaron diluciones de 0,5 a $2.048 \mu \mathrm{g} / \mathrm{ml}$ de amoxicilina, 8 a $512 \mu \mathrm{g} / \mathrm{ml}$ de sulbactam y $1 / 0,5$ a $518 / 256 \mu \mathrm{g} / \mathrm{ml}$ de amoxicilina-sulbactam

\section{Puntos de corte}

- Los puntos de corte utilizados en este estudio y de acuerdo a curvas de regresión previamente realizadas para E. coli y P. mirabilis ${ }^{4}$ se detallan en la Tabla 1.

- Se consideraron los puntos de corte a nivel urinario basados en las concentraciones de antimicrobianos alcanzadas en la orina luego de una dosis oral de amoxicilina-sulbactam de $500 / 500 \mathrm{mg}$ y en comprobaciones previas ${ }^{5}$ de los valores pico alcanzables por amoxicilina: $1.716 \mu \mathrm{g} / \mathrm{ml}(\mathrm{SD} \pm 744)$ y sulbactam: 1.850 $\mu \mathrm{g} / \mathrm{ml}(\mathrm{SD} \pm 922)$. En base a ello estimamos puntos de corte a "nivel urinario" para susceptibilidad: amoxicilina $<512 \mu \mathrm{g} / \mathrm{ml}$; sulbactam $<512 \mu \mathrm{g} / \mathrm{ml}$ y la combinación amoxicilinasulbactam $<512 / 256 \mu \mathrm{g} / \mathrm{ml}$.

Títulos inhibitorios de las orinas de voluntarios frente a aislamientos de $E$. coli. Las orinas de 12 voluntarios que dieron su consentimiento escrito ( 6 del sexo masculino; edad media 32 años, rango 23-39 años; peso medio $68 \mathrm{~kg}$ ), y que recibieron una única dosis oral de 500/500 $\mathrm{mg}$ de amoxicilina-sulbactam, fueron recolectadas antes de la administración de la asociación amoxicilina-sulbactam y a las 2, 4 y 6 h post administración. Se determinaron los TIO sobre 5 cepas de E. coli resistentes a amoxicilinasulbactam, tanto por el método de difusión como de dilución y sobre una cepa susceptible.

La técnica para la determinación del TIO fue la previamente descrita por nosotros ${ }^{4,6}$. En breve:

Tabla 1. Límites de corte para los métodos de difusión en agar y dilución seriada en agar utilizados en este estudio

\begin{tabular}{lccccccc}
\hline & \multicolumn{3}{c}{ Difusión $(\mathbf{m m})$} & \multicolumn{3}{c}{ CIM $(\boldsymbol{\mu g} / \mathbf{m l})$} \\
Antimicrobiano & $\mathbf{S}$ & $\mathbf{I}$ & $\mathbf{R}$ & $\mathbf{S}$ & $\mathbf{I}$ & \multicolumn{1}{c}{$\mathbf{R}$} \\
\hline Amoxicilina & $>16$ & $12-16$ & $<12$ & $<16$ & 16 & $>16$ \\
Amoxicilina-sulbactam & $>18$ & $14-18$ & $<14$ & $<16 / 8$ & $16 / 8$ & $>16 / 8$ \\
\hline
\end{tabular}

S: susceptible; I: intermedio; R: resistente 
las muestras de orina fueron conservadas a $-20^{\circ}$ $\mathrm{C}$ hasta su proceso. La orina pre-administración del antimicrobiano (basal) y las post-administración correspondientes a cada horario fueron descongeladas a temperatura ambiente y se efectuaron diluciones progresivas al 1/2 utilizando para ello la orina basal del propio voluntario; se obtuvieron así diluciones desde $1 / 1$ a $1 / 32$. Cada dilución de orina fue colocada en volúmenes de 5 $\mathrm{ml}$ en tubos estériles con tapa de rosca. A cada uno de ellos se les incorporó $0,1 \mathrm{ml}$ de una suspensión de cada cepa en estudio ajustada a la concentración de $10^{9} \mathrm{ufc} / \mathrm{ml}$. Se destaca que no se emplearon medios de cultivo como diluyente ya que la intención fue conocer los TIO en las condiciones propias de la orina vesical. Empleamos un alto inóculo de $10^{9} \mathrm{ufc} / \mathrm{ml}$ para remedar las altas concentraciones de E. coli que se alcanzan habitualmente en la orina vesical en las infecciones urinarias bajas luego de un adecuado tiempo de retención $(2 \text { horas })^{1,3,10}$ y por otra parte, desafiar a los antibacterianos con un aporte importante de $\beta$-lactamasas en las cepas productoras de las mismas. Se incubó un control de orina basal sin antimicrobiano y sin inóculo, para cada voluntario para demostrar su esterilidad, y por otra parte, tubos sin contener antimicrobiano de cada muestra horaria para demostrar la capacidad de desarrollo de la cepa inoculada en las orinas correspondientes. Todos los tubos se incubaron a $35^{\circ} \mathrm{C}$ durante 18 horas. $\mathrm{Al}$ cabo de ese tiempo observamos los tubos para determinar la turbidez por medio de un fotocolorímetro. El primer tubo en que no se observó desarrollo visible fue considerado el correspondiente al TIO. Por ejemplo, si no se observó desarrollo en el tubo correspondiente en el tubo $1 / 4$ pero fue observada a la dilución $1 / 8$, el valor $1 / 4$ fue considerado el TIO.

Concentraciones urinarias de amoxicilina y sulbactam. En las mismas orinas emitidas por los voluntarios se determinaron las concentraciones de amoxicilina y sulbactam por un método microbiológico que describiéramos recientemente y que comprobamos que no ofrecía variaciones con los datos obtenidos por cromatografía líquida de alta resolución ${ }^{7}$.

\section{Resultados}

En la Tabla 2 se muestra la prevalencia de especies responsables de IU bajas extrahospitalarias en los 10 centros participantes en un total de 1.000 cepas estudiadas. Los aislamientos de E. coli fueron predominantes en todos los países, como era de esperar, con un rango de 70 a $95 \%$ y un promedio de $82 \%$ que coincidió con estadísticas previas realizadas por el Comité de Antibióticos de la Sociedad Latinoamericana de Infectología Pediátrica ${ }^{11}$. Llamó la atención que en Chile el porcentaje de $14 \%$ de Proteus mirabilis aislados se apartó del rango encontrado en otros países (1 a 5\%) y que en el mismo sentido el porcentaje de K. pneumoniae aislados en el labo-

Tabla 2. Especies responsables de IU bajas en 1.000 urocultivos realizados en los 10 centros participantes

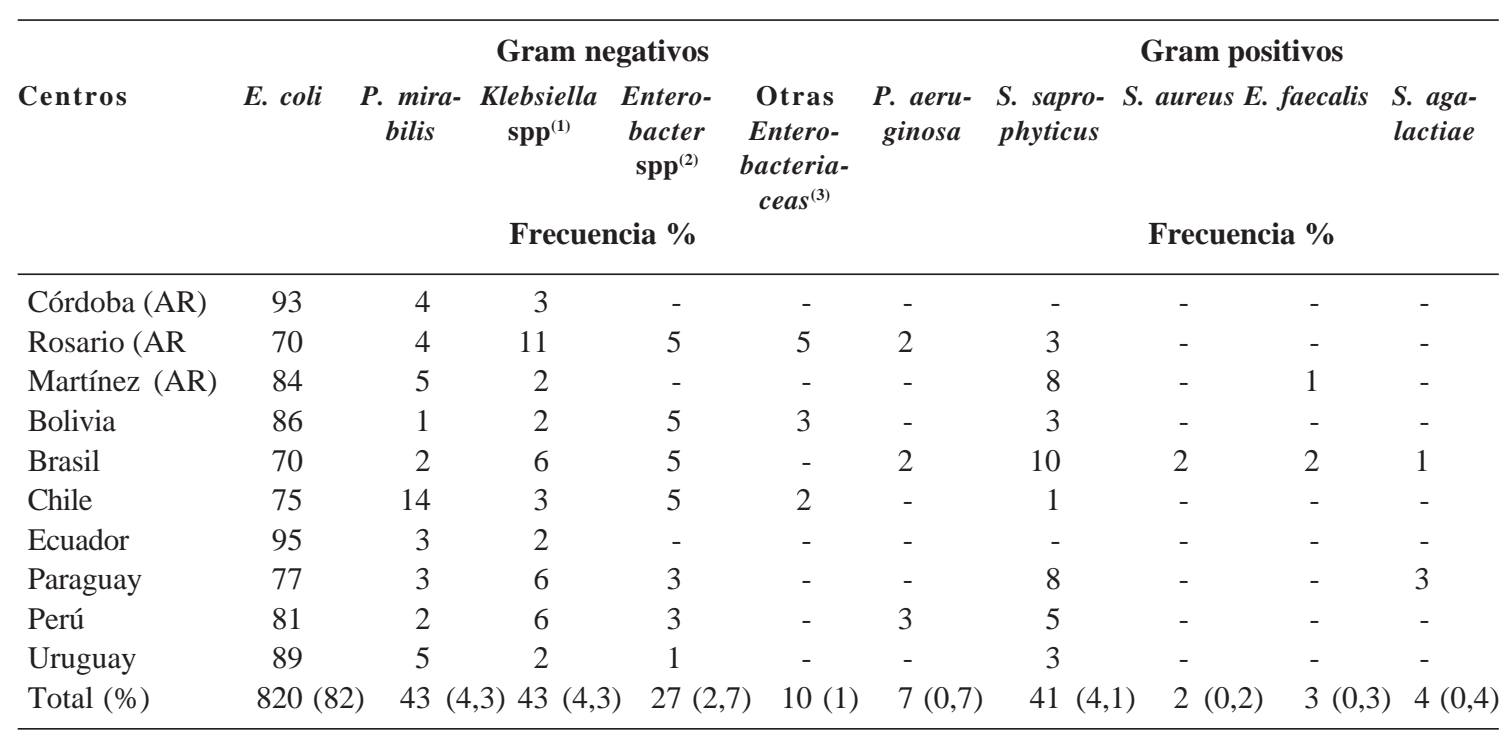

\footnotetext{
(1) K. pneumoniae $36 ;$ K. oxytoca 7

(2) E. cloacae 24; E. aerogenes 3

(3) Proteus vulgaris 3; Citrobacter freundii 2; Morganella morganii 2; Proteus penneri 3
} 
ratorio de la ciudad de Rosario de $11 \%$ también se apartó del rango de los demás centros $(2$ a $6 \%$ ). Dado que se trató de infecciones extrahospitalarias estas diferencias no pueden ser atribuibles a brotes. No se observaron diferencias en el aislamiento de bacterias Gram positivas.

En la Tabla 3 se detalla el porcentaje de susceptibilidad a amoxicilina y amoxicilina-sulbactam obtenido por el método de difusión en agar para 820 cepas de $E$. coli aisladas de pacientes con IU bajas extrahospitalarias. El rango de resistencia en $E$. coli a amoxicilina fue muy amplio (40 a $84,9 \%$ ), considerando que para la NCCLS las cepas que caen en la categoría intermedia son consideradas como susceptibles en las IU bajas $^{8,9}$. Los porcentajes de resistencia más bajos se observaron en Argentina, Brasil y Chile, los más elevados en Bolivia, Paraguay y Uruguay. La resistencia a amoxicilina-sulbactam también presentó un amplio rango (16 a 50,6\%) siendo los valores de resistencia más elevados los encontrados en Perú, Argentina y Uruguay y los menores en Paraguay y Chile.
En la Tabla 4 pueden observarse los valores de CIM de 100 cepas de E. coli resistentes a amoxicilina-sulbactam por el método de difusión obtenidas de los 10 centros sudamericanos, así como los valores observados con las cepas de control ATCC. Se destaca que el valor de la $\mathrm{CIM}_{50}$ de amoxicilina es considerablemente elevado $(>2.048 \mu \mathrm{g} / \mathrm{ml})$ y además que el valor de la $\mathrm{CIM}_{90}$ de sulbactam fue 10 veces menor que la concentración alcanzable por este compuesto en la orina vesical (Tabla 6).

En la Tabla 5 puede comprobarse los valores de TIO de los voluntarios luego de la administración de amoxicilina-sulbactam a una dosis oral de 500:500 mg sobre 60 cepas de E. coli seleccionadas con diferentes CIMs. Se comprueba que hasta la hora 6 se observaron TIO superiores a $1 / 2$ en todos los horarios para 55 de las 60 cepas estudiadas $(91,7 \%)$ y que la totalidad de las restantes cepas fueron inhibidas por la orina sin diluir o por diluciones hasta 1/4.

Finalmente, en la Tabla 6 se detallan las concentraciones medias de amoxicilina y de sulbactam

Tabla 3. Susceptibilidad de 820 cepas de Escherichia coli aisladas de pacientes con IUBNCC a amoxicilina y amoxicilina-sulbactam por el método de difusión en agar

\begin{tabular}{lccccccc}
\hline & & \multicolumn{3}{c}{ Amoxicilina } & \multicolumn{3}{c}{ Amoxicilina-sulbactam } \\
Centros & $\mathbf{N}^{\mathbf{0}}$ & $\begin{array}{c}\text { Susceptible } \\
\text { \% }\end{array}$ & $\begin{array}{c}\text { Intermedio } \\
\text { \% }\end{array}$ & $\begin{array}{c}\text { Resistente } \\
\text { \% }\end{array}$ & $\begin{array}{c}\text { Susceptible } \\
\text { \% }\end{array}$ & $\begin{array}{c}\text { Intermedio } \\
\text { \% }\end{array}$ & $\begin{array}{c}\text { Resistente } \\
\text { \% }\end{array}$ \\
\hline Córdoba (AR*) & 93 & 41,9 & 11,8 & 46,4 & 65,6 & 14 & 20,4 \\
Rosario (AR) & 70 & 47,1 & 12,9 & 40 & 54,3 & 10 & 35,7 \\
Martínez (AR) & 84 & 16,7 & 14,3 & 69 & 45,3 & 5,9 & 48,8 \\
Bolivia & 86 & 5,8 & 9,3 & 84,9 & 76,7 & 12,8 & 10,5 \\
Brasil & 79 & 18,6 & 32,9 & 48,5 & 50 & 31,4 & 18,6 \\
Chile & 75 & 45,3 & 9,4 & 45,3 & 57,3 & 26,7 & 16 \\
Ecuador & 95 & 16,8 & 25,3 & 57,9 & 41 & 29,5 & 29,5 \\
Paraguay & 77 & 9 & 17 & 74 & 58,4 & 27,3 & 14,3 \\
Perú & 81 & 17,3 & 14,8 & 67,9 & 34,6 & 14,8 & 50,6 \\
Uruguay & 89 & 7,9 & 21,3 & 70,8 & 44,9 & 19,2 & 35,9 \\
Total & 820 & 23,7 & 16,9 & 59,4 & 52,8 & 19,2 & 28 \\
\hline
\end{tabular}

* AR: Argentina

Tabla 4. CIM de amoxicilina, amoxicilina-sulbactam y sulbactam sobre 100 cepas de Escherichia coli resistentes a amoxicilina-sulbactam por el métodos de difusión en agar sobre cepas de $E$. coli ATCC

\begin{tabular}{lccccc}
\hline Antimicrobiano & Rango CIM & CIM $_{\mathbf{5 0}}^{*}$ & CIM $_{\mathbf{9 0}} \boldsymbol{*}^{*}$ & ATCC25922 $^{*}$ & ATCC35218 \\
\hline Amoxicilina & $32->2.048$ & $>2.048$ & $>2.048$ & 4 & 512 \\
Amoxicilina-sulbactam & $<8 / 4-512 / 256$ & $32 / 16$ & $256 / 128$ & $4 / 2$ & $16 / 8$ \\
Sulbactam & $32-256$ & 64 & 128 & 32 & 64 \\
\hline
\end{tabular}

$*(\mu \mathrm{g} / \mathrm{ml})$ 
Tabla 5. Títulos inhibitorios obtenidos en las muestras de orina de voluntarios sobre 60 cepas de $\boldsymbol{E}$. coli con diferentes niveles de susceptibilidad a amoxicilina-sulbactam

\begin{tabular}{lccccc}
\hline CIM $(\boldsymbol{\mu g} / \mathbf{m l})$ & $\mathbf{N}^{\mathbf{0}}$ & Hora 0 & Hora $\mathbf{2}^{*}$ & Hora $\mathbf{4}^{*}$ & Hora $\mathbf{6}^{*}$ \\
\hline $8 / 4$ & 10 & 0 & $>1 / 32$ & $1 / 32$ & $1 / 16$ \\
$16 / 8$ & 10 & 0 & $1 / 32$ & $1 / 16$ & $1 / 16$ \\
$32 / 16$ & 23 & 0 & $1 / 8$ & $1 / 2$ & $1 / 2$ \\
$64 / 32$ & 12 & 0 & $1 / 8$ & $1 / 4$ & $1 / 2$ \\
$128 / 64$ & 4 & 0 & $1 / 4$ & $1 / 2$ & $1 / 1$ \\
$256 / 128$ & 1 & 0 & $1 / 1$ & $1 / 1$ & $1 / 1$ \\
\hline
\end{tabular}

* Expresado en horas después de la administración de amoxicilina-sulbactam.

Tabla 6. Concentración urinaria de amoxicilina y amoxicilina-sulbactam en la orina de voluntarios luego de una dosis oral de amoxicilina-sulbactam (500/500 mg)

\begin{tabular}{ccc}
\hline Hora & Amoxicilina & Sulbactam \\
\hline $2 \mathrm{~h}$ & $1.414^{*}$ & $1.904^{*}$ \\
$4 \mathrm{~h}$ & 691 & 1.257 \\
$6 \mathrm{~h}$ & 462 & 641 \\
\hline
\end{tabular}

$*(\mu \mathrm{g} / \mathrm{ml})$

que se hallaron en la orina de los voluntarios luego de recibir la dosis mencionada. Como puede comprobarse, los valores alcanzados hasta las 6 horas superan la concentración correspondiente a la máxima CIM obtenida para sulbactam (128 $\mu \mathrm{g} / \mathrm{ml})$.

\section{Discusión}

Nuestro estudio muestra que E. coli fue responsable de más de $70 \%$ de los aislamientos de orina en casos de IUBNCC en ambos sexos en los centros de los países sudamericanos que participaron del estudio. $P$. mirabilis y $K$. pneumoniae (4,3\% cada uno); Staphylococcus saprophyticus $(4,1 \%)$ y otras especies $(5,2 \%)$ constituyeron el resto de los aislamientos. Llamamos la atención sobre la baja incidencia de Enterococcus; ello fue probablemente debido a que solicitamos a los investigadores que excluye cepas de enterococos de orinas que hubieran sido refrigeradas por más de una hora, ya que, como es bien conocido, los enterococos son capaces de reproducirse a $4^{\circ} \mathrm{C}$.

El número de cepas de E. coli estudiadas (820) es realmente significativo. Sin embargo, puede argumentarse que habiendo participado un solo centro en la mayoría de los países, los datos no representan necesariamente una realidad nacional. Si bien a nivel continental los porcentajes coinciden con estudios previos realizados por SLIPE $^{11}$.

La resistencia observada a amoxicilinasulbactam en E. coli fue de $28 \%$ en promedio en base a los puntos de corte de la NCCLS ${ }^{9}$. Esta resistencia, es aplicable a infecciones extraurinarias y es debida a la incapacidad de los inhibidores de $\beta$-lactamasas (sulbactam, ácido clavulánico, tazobactam) de efectuar un bloqueo suicida irreversible de la $\beta$-lactamasa TEM-1 prevalente en $E$. coli cuando ésta se hiperproduce ${ }^{2}$. La hiperproducción de TEM-1 es frecuente en nuestro medio $^{5}$ (R. Zemelmann, comunicación personal) y los valores de CIM para amoxicilina en tales casos superan los $2.000 \mu \mathrm{g} / \mathrm{ml}$, lo que impide que los inhibidores de $\beta$-lactamasas puedan cumplir su cometido de reducir la CIM de amoxicilina en la combinación con el inhibidor, a concentraciones que estén por debajo de las alcanzables en plasma. Sin embargo, es consenso entre los pediatras $^{3,14}$ que el uso de aminopenicilinas unidas a inhibidores de $\beta$-lactamasas, de cefalosporinas de $1^{a}$ generación y de nitrofurantoína, son clínicamente efectivas en IUBNCC a pesar de la resistencia anunciada en los antibiogramas ${ }^{3}$. Esta discrepancia entre la efectividad clínica en IUBNCC y los resultados de los antibiogramas se debe a que en éstos no se consideran los niveles de antibacteriano alcanzables en la orina en relación a la concentración de antibacterianos que inhiben a las cepas de E. coli u otros microorganismos. Es un problema similar al de las discrepancias, recién resueltas en este siglo, entre la actividad de amoxicilina en infecciones respiratorias y la llamada resistencia a penicilina que es aplicable a infecciones del sistema nervioso central. Para estas diferencias relacionadas a los puntos de corte que se usan en los antibiogramas el CDC acuño el término "the in vivo-in vitro paradox"15 que bien puede aplicarse en nuestro caso. 
Los valores de CIM de amoxicilina-sulbactam observados en 100 cepas de E. coli consideradas resistentes por el método de discos, mostraron que los valores de $\mathrm{CIM}_{90}$ para las mismas fue de $256 / 128 \mu \mathrm{g} / \mathrm{ml}$ para amoxicilina-sulbactam y $128 \mu \mathrm{g} / \mathrm{ml}$ en sulbactam. Es de destacar que ambas concentraciones se encuentran francamente debajo de las que se obtienen en la orina luego de la administración de una dosis oral de 500:500 de amoxicilina-sulbactam. Por lo tanto, considerando los niveles urinarios de amoxicilina y de amoxicilina-sulbactam obtenidos luego de dicha dosis, todas las cepas aisladas de infecciones urinarias bajas no complicadas debieran considerarse como probablemente "susceptibles a niveles urinarios" de amoxicilina-sulbactam. Como demostramos previamente estas mismas consideraciones serian aplicables a combinaciones de amoxicilina con otros inhibidores de $\beta$-lactamasas tales como amoxicilina-ácido clavulánico ${ }^{6}$.

La actividad de sulbactam como único antibacteriano sobre E. coli es probablemente debida a la acción de este compuesto bloqueando a ciertas PBP, lo que ocurre a concentraciones superiores a las que se alcanzan en suero (D. Livermore, comunicación personal).

Los TIO obtenidos en la orina de voluntarios sobre 50 cepas resistentes a amoxicilina-sulbactam por el método de discos, demuestran que ni los resultados denominados "intermedio" ni los "resistentes" por el método de discos según la NCCLS, son capaces de predecir posibles fallas terapéuticas en pacientes tratados con fármacos que alcanzan altos niveles urinarios tales como amoxicilina-sulbactam, lo que ha sido también demostrado con otros antibacterianos tales como cefalosporinas de $1^{\mathrm{a}}$ generación ${ }^{12,13}$.

En conclusión, estos resultados proporcionan una base farmacodinámica para explicar el éxito observado con el uso de amoxicilina-sulbactam en el tratamiento de IU bajas no complicadas no bacteriemicas, aún en los casos en los que el antibiograma supone resistencia y, por lo tanto, probable ineficacia clínica.

\section{Resumen}

Previamente, habíamos demostrado la actividad que poseen amoxicilina-sulbactam y sulbactam solo frente a Escherichia coli en la orina. Realizamos un estudio para determinar la etiología de las infecciones urinarias bajas no complicadas de la comunidad (IUBNCC) en Sudamérica. Participaron 10 laboratorios de 8 países sudamericanos. Cada laboratorio envió al centro coordinador
(CEA, Bs. Aires) los resultados de susceptibilidad a amoxicilina y amoxicilina-sulbactam por el método de discos en 100 aislamientos consecutivos obtenidos de pacientes de 3 a 70 años con IUBNCC y además remitieron 20 cepas de $E$. coli consecutivas consideradas resistentes a amoxicilina por el método de discos. En el CEA se comprobó la CIM de amoxicilina, amoxicilinasulbactam (2:1) y sulbactam solo; se determinó el título inhibitorio de la orina (TIO) en 12 voluntarios que recibieron una dosis oral de 500:500 $\mathrm{mg}$ de amoxicilina-sulbactam. Las orinas se recolectaron a las 0 a 2, 2 a 4 y 4 a 6 h luego de la administración de amoxicilina-sulbactam y los TIO se verificaron sobre 5 cepas de E. coli resistentes (R) y 1 cepa sensible ( $\mathrm{S}$ ) a amoxicilinasulbactam seleccionadas con diferentes CIM, entre las recibidas de cada centro participante; se determinaron las concentraciones de amoxicilina y sulbactam en la orina por un método microbiológico. Resultados: E. coli fue predominante 820/ $1.000(82 \%) ;$ P. mirabilis y $K$. pneumoniae $(4,3 \%$ ambas); $S$. saprophyticus $(4,1 \%)$ y otros $(5,3 \%)$. Susceptibilidad por discos en E. coli: $59,4 \% \mathrm{R}$ a amoxicilina; $16,9 \%$ intermedias (I) y $23,7 \% \mathrm{~S}$ y para amoxicilina-sulbactam $28 \% \mathrm{R}$, 19,2\% I y $52,8 \%$ S. Determinación de CIM: se estudiaron 102 E. coli, R a amoxicilina-sulbactam, las $\mathrm{CIM}_{90}(\mu \mathrm{g} / \mathrm{ml})$ fueron: amoxicilina > 2.048; amoxicilina-sulbactam: $256 / 128$ y sulbactam solo, 128. TIO: Variaron desde $>1 / 32$ a las $2 \mathrm{~h} ; 1 / 16-$ $1 / 4$ a las $4 \mathrm{~h} \mathrm{y} 1 / 4-1 / 2$ a las $6 \mathrm{~h}$ para todas las cepas estudiadas. Niveles de ATB en orina $(\mu \mathrm{g} /$ $\mathrm{ml}$ ): amoxicilina y sulbactam respectivamente a las 2 h: 1.414 y 1.904; a las 4 h: 691 y 1.257 y a las 6 h: 462 y 641. Nuestros resultados confirman el predominio de E. coli en IUBNCC en Sudamérica y explican las discrepancias entre la resistencia supuesta por el método de discos y los éxitos clínicos logrados con amoxicilinasulbactam en IUBNCC.

\section{Bibliografía}

1.- Nicolle L E. Measurement and significance of antibiotic activity in the urine. In: Lorian V. Antibiotics in Laboratory Medicine. $4^{\text {th }}$ Ed. Baltimore, M R, USA Williams \& Wilkins 1996; 793-812.

2.- Livermore D M. $\beta$-lactamases in laboratory and clinical resistance. Clin Microbiol Rev 1995; 8: 557-84.

3.- Casellas J M. Antibióticos y antibiogramas en infecciones urinarias pediátricas adquiridas en la comunidad. Arch Latin Nefr Ped 2001; 1 (1): 17-34.

4.- Casellas J M, Arenoso H, Soutric J et al. Estudio comparativo in vitro de 3 asociaciones de inhibidores suicidas de $\beta$-lactamasas con aminopenicilinas. Rev Esp Quimioter 1993; 6: 289-97. 
5.- Bantar C, Nicola F, Arenoso H et al. Pharmacokinetics and pharmacodynamics of amoxicillinsulbactam, a novel aminopenicillin- $\beta$-lactamase inhibitor combination, against Escherichia coli. Antimicrob Agents Chemother 1999; 43: 1503-4.

6.- Casellas J M, Visser M, Tomè G, Cohen H, Soutric J, Arenoso H. Erradicación de Escherichia coli de la orina vesical por amoxicilina-sulbactam. Actividad intrínseca del inhibidor. Enferm Infecc Microbiol Clin 2001; 19: 206-10.

7.- Murray P, Baron E, Pfaller M, Tenover F, Yolken R. Manual of Clinical Microbiology. $7^{\text {th }}$ Ed. American Society for Microbiology. Washington DC, 1999.

8.- National Committee for Laboratory Standards. 22: M100-S2. Wayne PE, 2002.

9.- National Committee for Laboratory Standards. 22: M2A7. Wayne P E, 2002.

10.- Virtanen S, Oksanen T, Peltonen T. Colony counts and the diagnosis of urinary tract infection in infants and children. Annis Pediat 1982; 8: 269-74.

11.- Casellas, Prado V, Farinati A. Mesa redonda Datos de
SLIPE (El Salvador)

12.- Casellas J M, Exeni C, Berardi C et al. Estudio clínico y microbiológico acerca de la eficacia de cefalexina a dos dosis diarias comparada con cotrimoxazol en el tratamiento de infecciones urinarias bajas no complicadas adquiridas en la comunidad en niñas. Arch Arg Ped 2003; 101 (en prensa).

13.- Casellas J M, Exeni C, Exeni R et al. Clinical success when treating lower non complicated pediatric urinary tract infections with cefadroxil are not in accordance with the results of agar diffusion susceptibility tests. The Brazilian J Infec Dis 2003; 7 (en prensa).

14.- Infección urinaria. En Libro Azul de Infectología Pediátrica. $2^{\mathrm{a}}$ Edición. p. 107-120. Comité Nacional de Infectología. Sociedad Argentina de Pediatría. Buenos Aires. 2002.

15.- Heffelfinger J D, Dowell S F, Jorgensen J H et al. Management of community-acquired pneumonia in the era of pneumococcal resístance: From the drugresistant $S$. pneumoniae therapeutic working group. Arch Intern Med 2000 160: 1399-408.

Correspondencia a:

José María Casellas

E-mail: casellasjm@cpsarg.com 